\title{
Dynamic Response Analysis of an Asymmetric Coupled Vehicle-Track System Generated by Voided Elastic Two-Block Sleeper
}

\author{
Zhenxing $\mathrm{He}^{1}$ and Xinwen Yang ${ }^{2}$ \\ ${ }^{1}$ Metro Design and Research Institute of China Railway Eryuan Engineering Group Co. Ltd., Chengdu 610031, China \\ ${ }^{2}$ Key Laboratory of Road and Traffic Engineering of the Ministry of Education, Tongji University, Shanghai 201804, China \\ Correspondence should be addressed to Xinwen Yang; yangxinwen0603@163.com
}

Received 13 July 2015; Accepted 6 September 2015

Academic Editor: Nuno M. Maia

Copyright (C) 2016 Z. He and X. Yang. This is an open access article distributed under the Creative Commons Attribution License, which permits unrestricted use, distribution, and reproduction in any medium, provided the original work is properly cited.

Based on vehicle-track coupled dynamic theory, a three-dimensional asymmetric vehicle-track coupling vibration model is developed to investigate the effect of voided elastic two-block sleepers on vehicle and track system dynamic responses. For the vehicle system, one car body, two frames, and four wheel sets are assumed to be rigid, with 35 degrees of freedom (DOF). For the track system, the rails and the concrete two-block sleepers are the main vibration components. The rails are modelled as Timoshenko beams, and the concrete two-block sleepers are assumed to be rigid mass with vertical and lateral movement. The pads under the rails and the rubber boots under the sleepers provide greater vertical and lateral elasticity for the track. The Hertz nonlinear elastic contact theory is used to calculate the normal wheel/rail force. The wheel/rail tangent creep force is first calculated using Kalker's linear creep theory and then modified by the Shen-Hedrick-Elkins theory. The results show that the asymmetric voided elastic two-block sleepers have greater effects on the dynamic responses for fasteners and sleepers than on the car body and the wheel/rail forces under measured geometric irregularity and random irregularity. Two or more voided sleepers will greatly affect the vehicle running safety.

\section{Introduction}

In China, reinforced concrete monolithic track beds with two-block sleeper are widely used in the urban transit railway. For vibration reduction, the concrete two-block sleeper is usually placed into rubber boots to improve the elasticity of the track. Due to the adjusting error in the track construction, aging of the rubber pads, and the track beds being immersed in water during long-term operation, elastic two-block sleeper becomes void and causes track stiffness irregularity, such as the sudden presence of hard spots in the rail support, as shown in Figure 1.

Voided sleepers are commonly found in ballasted tracks $[1,2]$, causing the sleeper to crush and the service life of the rail to be shortened. Recent studies [3, 4] have investigated the dynamic problems caused by voided long sleepers in ballasted tracks. Based on vehicle-track coupled dynamics theory, a two-dimensional model was used to analyse the influence of voided sleepers or the sleeper span on the vertical displacements of the rails, the sleeper's pressures, and the vertical wheel/rail forces. In addition, the effect of "hard spots" under the rail, induced by the supports of long sleepers being too high [5-9], on the change in the wheel/rail force and the pressure on sleepers was studied by using a threedimensional vehicle-track coupling dynamics model, and the results showed that the interaction force between the rail and the sleeper is sensitive to stiffness irregularity [10]. A threedimensional model was also used to analyse the influence of a voided sleeper on the dynamic responses of an adjacent sleeper in the ballasted track. When the sleeper is partially or completely voided, the maximum bending moment acting on the sleeper moves from the section under the rail to the middle section, from which it is easy to induce transverse cracks in the upper part of the middle section [11].

In the above research, it is obvious that the twodimensional or three-dimensional vehicle-track coupling 


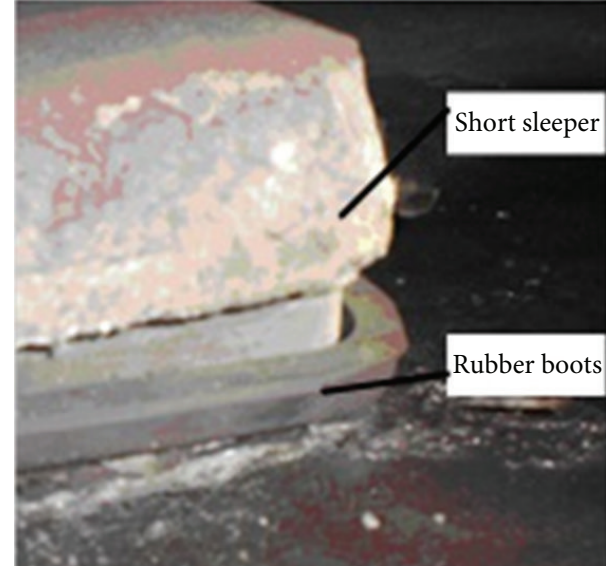

Figure 1: Voided elastic two-block sleeper.

models were mainly established to analyse the influence of voided sleepers on the load increment of the adjacent sleepers in ballasted track with long sleepers. However, the influences of voided two-block sleeper on the vehicle and track system have been hardly studied in the unballasted track of the urban transit railway. In addition, once one or more sleepers are voided, the support system under the rails will become asymmetric, which causes more dynamic problems in the vehicle and track system. To solve the problems caused by voided sleepers, based on vehicle-track coupled dynamic theory, a three-dimensional asymmetric dynamic model is developed to study the coupling effect between the vehicle system and the track system with elastic two-block sleeper monolithic beds under measured geometric irregularity and random irregularity. Through numerical simulation, the influence of a sudden change in support stiffness of the rail on the dynamic responses for the vehicle system and track system is also probed.

\section{Dynamic Model}

2.1. Model of Vehicle-Track Coupling System. A threedimensional asymmetric vehicle-track coupling dynamic model [5] is shown in Figures 2 and 3. For the vehicle system, the car body, the frame, and the wheel sets, which are connected to each other with springs and dampers, are assumed to be rigid. The wheel set and the frame are connected by the primary suspension, while the body is supported on the frame through the secondary suspension. The primary suspension system includes the axle box springs, which have stiffness in three directions; the axle box positioning equipment, which has lateral stiffness; and the vertical primary dampers outside the axle box springs, which have lateral damping. The secondary suspension system includes the springs, which have stiffness and damping in three directions; the rubber blocks, which function as the lateral stop; the antiyaw dampers; the lateral dampers; and the vertical dampers. Therefore, the total degrees of freedom (DOF) of the vehicle model are 35, including lateral, vertical, roll, pitch, and yaw motions of the vehicle component (one car body, two frames, and four wheel sets). It should be noted that the longitudinal motions of the vehicle are ignored.

In Figures 2 and $3, Y, Z, \phi, \beta$, and $\psi$ represent the lateral, vertical, roll, pitch, and yaw motions of the vehicle system, respectively; $M_{c}, M_{t}$, and $M_{w}$ are the mass of the car body, the frame, and the wheel set, respectively; $I_{w x}$ and $I_{w y}$ are, respectively, rolling and rotating moment of inertia of the wheel set; $I_{t x}$ and $I_{t y}$ are rolling and nodding moment of inertia of the frame, respectively; $I_{c x}$ and $I_{c y}$ are rolling and nodding moment of inertia of the car body, respectively; $K_{s y}$ and $K_{s z}$ are lateral and vertical stiffness of the second suspension at one side of the frame, respectively; $C_{s y}$ and $C_{s z}$ are lateral and vertical damping of the second suspension at one side of the frame, respectively; $K_{r x}$ is the antirolling stiffness; $K_{p y}$ and $K_{p z}$ are lateral and vertical stiffness of each primary suspension, respectively; and $C_{p y}$ and $C_{p z}$ are lateral and vertical damping of each primary suspension, respectively.

The track system is modelled according to the elastic twoblock sleeper monolithic track bed. The rails and the concrete two-block sleeper are the main vibration components. The rails are modelled as Timoshenko beams, and the concrete two-block sleepers are assumed to be rigid mass, which have vertical and lateral movement. The pads under the rails and the rubber boots under the sleepers give the track greater vertical and lateral elasticity so that the track reduces the impact of the train loads. In Figures 2 and 3, $m_{r}$ is the mass of the rail; $E I$ is the bending stiffness of the rail; $M_{s}$ is the mass of the sleeper; $K_{p v}$ and $K_{p h}$ are the vertical and lateral stiffness of a fastener and a pad, respectively; $C_{p v}$ and $C_{p h}$ are, respectively, the vertical and lateral damping of a fastener and a pad, respectively; $K_{b v}$ and $K_{b h}$ are the vertical and lateral stiffness of a rubber boot under the sleeper, respectively; $C_{b v}$ and $C_{b h}$ are the vertical and lateral damping of a rubber boot under the sleeper, respectively.

The wheel/rail interaction is the connection between the vehicle system and the track system. The rolling contact of the wheel and the rail generates the necessary conditions for a railway vehicle to run stably on the track. The rolling contact can be described by the kinematic constraints (geometric contacts) as well as the dynamic constraints (physical contacts), which are, respectively, referred to as the contact geometry relationship and contact forces between the wheel and the rail. In the present paper, the geometric problem of wheel/rail contact is solved spatially and evaluated online. The numerical method for the contact geometry calculation is discussed in detail [5].

The calculation of wheel/rail contact forces includes a normal model and a tangent model. The normal model, which characterizes the relationship law of the normal load and deformation between the wheel and the rail, is described by a Hertzian nonlinear contact theory [5]. The model by Shen et al. [12] is used as the tangent model determining the relationship between the creepages and the total creep forces of the wheel/rail. In the calculation of the tangent creep forces by Shen's model, the creepages of the wheel and rail should be previously calculated in the transient analysis of the vehicle-track coupling dynamics. The creepage calculation is concerned with the parameters of the contact geometry of 


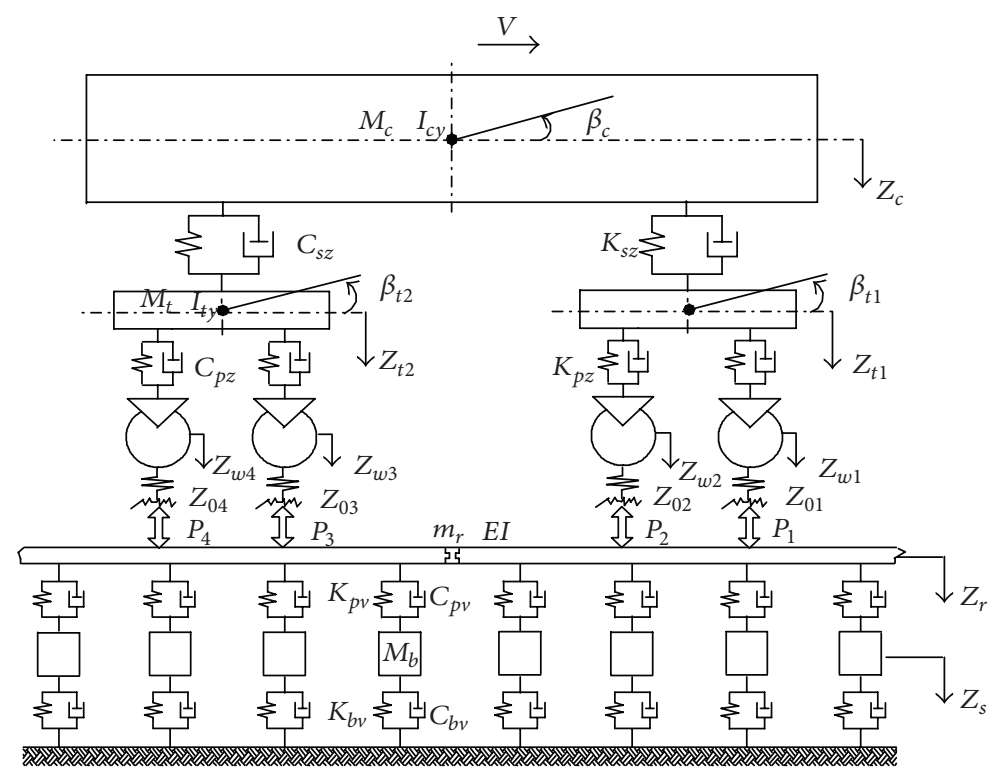

FIGURE 2: Lateral view of the dynamic model.

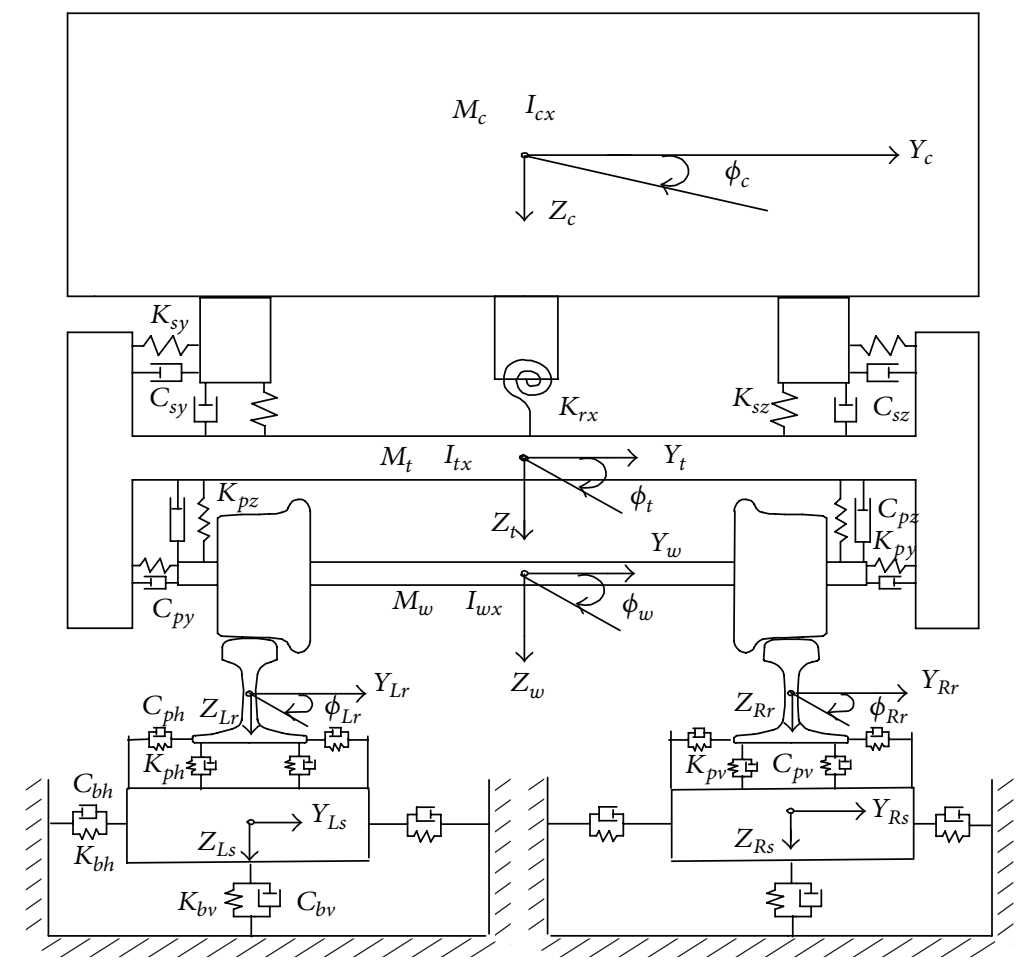

FIgURE 3: Side view of the dynamic model.

the wheel/rail, such as instant circle radii of the wheels and contact angles between the wheels and the rails.

\subsection{Equations of Motion of the System}

2.2.1. Equations of Motion of a Metro Vehicle. For the vehicle system, by using the coordinate system moving along the track with vehicle speed, the equations of motion of the metro vehicle can be easily derived according to D'Alembert's principle, which can be described in the form of second-order differential equations in time domain:

$$
\begin{gathered}
M_{V} A_{V}+C_{V}\left(V_{V}\right) V_{V}+K_{V}\left(X_{V}\right) X_{V} \\
=F_{V}\left(X_{V}, V_{V}, X_{T}, V_{T}\right)+F_{\mathrm{EXT}},
\end{gathered}
$$




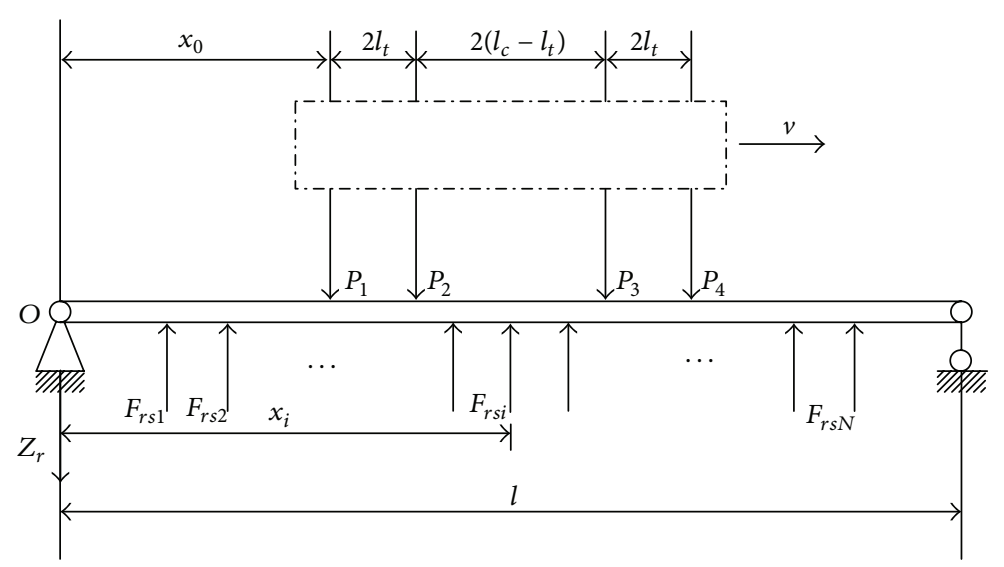

FIGURE 4: Sketch of the forces acting on a rail.

where $X_{V}, V_{V}$, and $A_{V}$ are the vectors of displacement, velocity, and acceleration of the vehicle subsystem, respectively; $M_{V}$ is the mass matrix of the vehicle; $C_{V}\left(V_{V}\right)$ and $K_{V}\left(X_{V}\right)$ are the damping and the stiffness matrices which depend on the state of the vehicle system to describe the nonlinearities of the suspensions; $X_{T}$ and $V_{T}$ are the vectors of displacement and velocity of the track system; $F_{V}\left(X_{V}, V_{V}, X_{T}, V_{T}\right)$ is the load vector acting on the vehicle system, composed of the nonlinear wheel/rail contact forces which are determined by the wheel-track coupling model and depend on $X_{V}, V_{V}, X_{T}$, and $V_{T}$; and $F_{\mathrm{EXT}}$ is the external forces, including wheel/rail contact force, and gravitational forces and centripetal forces resulting from the vehicle running through the curve track.

2.2.2. Equations of Motion of the Track. In the track system, the rail is modelled as a Timoshenko beam. The forces acting on the rail are shown in Figure 4.

(1) The Rail. As for a rail on the right side of the track, the equation of vertical motion of the rail is

$$
\begin{aligned}
& m_{r} \frac{\partial^{2} Z_{r}(x, t)}{\partial t^{2}}+\kappa_{z} G A\left[\frac{\partial \psi_{z r}(x, t)}{\partial x}-\frac{\partial^{2} Z_{r}(x, t)}{\partial x^{2}}\right] \\
& =-\sum_{i=1}^{N_{s}} F_{R r V i}(t) \delta\left(x-x_{F i}\right)+\sum_{j=1}^{N_{w}} P_{V j}(t) \delta\left(x-x_{P j}\right), \\
& \rho_{r} I_{y} \frac{\partial^{2} \psi_{z r}(x, t)}{\partial t^{2}}+\kappa_{z} G A\left[\psi_{z r}(x, t)-\frac{\partial Z_{r}(x, t)}{\partial x}\right] \\
& -E I_{y} \frac{\partial^{2} \psi_{z r}(x, t)}{\partial x^{2}}=0 .
\end{aligned}
$$

The equation of lateral motion is

$$
\begin{aligned}
& m_{r} \frac{\partial^{2} Y_{r}(x, t)}{\partial t^{2}}+\kappa_{y} G A\left[\frac{\partial \psi_{y r}(x, t)}{\partial x}-\frac{\partial^{2} Y_{r}(x, t)}{\partial x^{2}}\right] \\
& =-\sum_{i=1}^{N_{s}} F_{R r L i}(t) \delta\left(x-x_{F i}\right)+\sum_{j=1}^{N_{w}} P_{L j}(t) \delta\left(x-x_{P j}\right),
\end{aligned}
$$

$$
\begin{aligned}
& \rho_{r} I_{z} \frac{\partial^{2} \psi_{y r}(x, t)}{\partial t^{2}}+\kappa_{y} G A\left[\psi_{y r}(x, t)-\frac{\partial Y_{r}(x, t)}{\partial x}\right] \\
& -E I_{z} \frac{\partial^{2} \psi_{y r}(x, t)}{\partial x^{2}}=0 .
\end{aligned}
$$

The equation of vertical motion is given by

$$
\begin{aligned}
\rho_{r} & I_{r 0} \frac{\partial^{2} \phi_{r}(x, t)}{\partial t^{2}}+G I_{t} \frac{\partial^{2} \phi_{r}(x, t)}{\partial x^{2}} \\
& =-\sum_{i=1}^{N_{s}} M_{r T i}(t) \delta\left(x-x_{F i}\right)+\sum_{j=1}^{N_{w}} Q_{T j}(t) \delta\left(x-x_{P j}\right),
\end{aligned}
$$

where $Y_{r}, Z_{r}$, and $\phi_{r}$ are, respectively, the lateral, vertical, and torsion deflections of the rail, $\psi_{y r}$ and $\psi_{z r}$ are the slope of the deflection curve of the rail with respect to $z$ - and $y$-axes, $\rho_{r}$ is the rail density, $A$ is the area of the rail cross section, $m_{r}=\rho_{r} A, G$ is the shear modulus of the rail, $E$ is Young's modulus of the rail material, $I_{y}$ and $I_{z}$ are, respectively, the second moment of area of the rail cross section about the $y$ and $z$-axes, $I_{r 0}$ is the polar moment of inertia of the rail cross section, $\kappa_{y}=0.4057$ and $\kappa_{z}=0.5329$, denoting, respectively, the lateral and vertical shear coefficients, which are obtained through the calculation of China's rail cross section CN60 using the finite element method software ANSYS, and $P_{V j}(t)$ and $P_{L j}(t)$ are, respectively, the vertical and lateral wheel/rail forces between wheel $j$ and the rail, which changes with the vehicle speed $v, M_{r T i}$ and $Q_{T j}$ are the force moment on the rail, $\delta(x)$ is the Dirac delta function, $x_{F i}$ is the coordinate of sleeper $i, x_{p j}$ is the coordinate of wheel $j$, and $N_{w}$ and $N_{s}$ are the numbers of wheel sets and sleepers within the analysed rail. The subscript $i$ indicates sleeper $i$, and $j$ wheel $j . F_{R r V i}(t)$ and $F_{R r L i}(t)\left(i=1 \sim N_{s}\right)$ are the vertical and lateral forces between the rail and the $i$ th sleeper, respectively:

$$
\begin{aligned}
F_{R r V i}(t)= & K_{p v i}\left[Z_{r}\left(x_{F i}, t\right)-Z_{b}\left(x_{F i}, t\right)\right] \\
& +C_{p v i}\left[\dot{Z}_{r}\left(x_{F i}, t\right)-\dot{Z}_{b}\left(x_{F i}, t\right)\right]
\end{aligned}
$$




$$
\begin{aligned}
F_{R r L i}(t)= & K_{p h i}\left[Y_{r}\left(x_{F i}, t\right)-Y_{b}\left(x_{F i}, t\right)\right] \\
& +C_{p h i}\left[\dot{Y}_{r}\left(x_{F i}, t\right)-\dot{Y}_{b}\left(x_{F i}, t\right)\right] .
\end{aligned}
$$

The displacements of the rail are written as

$$
\begin{aligned}
Z_{r}(x, t) & =\sum_{k=1}^{\mathrm{NMR}} Z_{k}(x) q_{z k}(t), \\
\psi_{z r}(x, t) & =\sum_{k=1}^{\mathrm{NMR}} \Psi_{z k}(x) w_{z k}(t), \\
Y_{r}(x, t) & =\sum_{k=1}^{\mathrm{NMR}} Y_{k}(x) q_{y k}(t), \\
\psi_{y r}(x, t) & =\sum_{k=1}^{\mathrm{NMR}} \Psi_{y k}(x) w_{y k}(t), \\
\phi_{r}(x, t) & =\sum_{k=1}^{\mathrm{NMRT}} \Theta_{k}(x) q_{t k}(t),
\end{aligned}
$$

where $q_{z k}(t), w_{z k}, q_{y k}(t), w_{y k}$, and $q_{t k}(t)$ are the modal displacement (coordinates) of the rail and NMR are the number of modes considered for the rail $(\mathrm{NMR}=100)$. The frequencies of the modal shape functions of the rail are up to $1 \mathrm{kHz}$. The modal shape functions of the rail are given below:

$$
\begin{aligned}
Z_{k}(x) & =\sqrt{\frac{2}{m_{r} l}} \sin \frac{k \pi x}{l}, \\
\Psi_{z k}(x) & =\sqrt{\frac{2}{\rho_{r} I_{z} l}} \cos \frac{k \pi x}{l}, \\
Y_{k}(x) & =\sqrt{\frac{2}{m_{r} l}} \sin \frac{k \pi x}{l}, \\
\Psi_{y k}(x) & =\sqrt{\frac{2}{\rho_{r} I_{y} l}} \cos \frac{k \pi x}{l}, \\
\Theta_{k}(x) & =\sqrt{\frac{2}{\rho_{r} I_{0} l}} \sin \frac{k \pi x}{l},
\end{aligned}
$$

where $l$ is the calculation length of the rail.

By applying Ritz's method, the fourth-order partial differential equation of motion of the rails then simplified to a series of second-order ordinary differential equations in terms of the time coordinate, and $q_{z k}(t), w_{z k}(t), q_{y k}(t), w_{y k}(t)$, and $q_{t k}(t)$ are

$$
\begin{aligned}
& \ddot{q}_{z k}(t)+\frac{\kappa_{z} G}{\rho_{r}}\left(\frac{k \pi}{l}\right)^{2} q_{z k}(t) \\
& +\frac{\kappa_{z} G k \pi}{\rho_{r} l} \sqrt{\frac{A}{I_{z}}} w_{z k}(t)=-\sum_{i=1}^{N_{s}} F_{R r V i} Z_{k}\left(x_{F i}\right)
\end{aligned}
$$

$$
+\sum_{j=1}^{N_{w}} P_{V j} Z_{k}\left(x_{P j}\right)
$$

$$
\begin{aligned}
& \ddot{w}_{z k}(t)+\left[\frac{\kappa_{z} G A}{\rho_{r} I_{z}}+\frac{E}{\rho_{r}}\left(\frac{k \pi}{l}\right)^{2}\right] w_{z k}(t) \\
& -\frac{\kappa_{z} G k \pi}{\rho_{r} l} \sqrt{\frac{A}{I_{z}}} q_{z k}(t)=0 \quad(k=1 \sim \mathrm{NMR}),
\end{aligned}
$$$$
\ddot{q}_{y k}(t)+\frac{\kappa_{y} G}{\rho_{r}}\left(\frac{k \pi}{l}\right)^{2} q_{y k}(t)
$$$$
+\frac{\kappa_{y} G k \pi}{\rho_{r} l} \sqrt{\frac{A}{I_{y}}} w_{y k}(t)=-\sum_{i=1}^{N_{s}} F_{R r L i} Y_{k}\left(x_{F i}\right)
$$$$
+\sum_{j=1}^{N_{w}} P_{L j} Y_{k}\left(x_{P j}\right)
$$

$$
\begin{aligned}
& \ddot{w}_{y k}(t)+\left[\frac{\kappa_{y} G A}{\rho_{r} I_{y}}+\frac{E}{\rho_{r}}\left(\frac{k \pi}{l}\right)^{2}\right] w_{y k}(t) \\
& -\frac{\kappa_{y} G k \pi}{\rho_{r} l} \sqrt{\frac{A}{I_{y}}} q_{y k}(t)=0 \quad(k=1 \sim \mathrm{NMR})
\end{aligned}
$$

$$
\begin{gathered}
\ddot{q}_{t k}(t)+\frac{G I_{r t}}{\rho_{r} I_{r 0}}\left(\frac{k \pi}{l}\right)^{2} q_{t k}(t)=-\sum_{i=1}^{N_{s}} F_{r s T i} \Theta_{k}\left(x_{F i}\right) \\
+\sum_{j=1}^{N_{w}} P_{T j} \Theta_{k}\left(x_{P j}\right) \quad(k=1 \sim \mathrm{NMR}) .
\end{gathered}
$$

(2) The Two-Block Sleeper. For concrete two-block sleeper, the relationship between the forces is shown in Figure 5. Based on the interaction between the rail and the sleeper, the system equation is obtained.

The equation of the vertical motion of the left block is

$$
M_{b} \ddot{Z}_{L b i}=F_{L r V i}-F_{L s V i} \text {. }
$$

The equation of the lateral motion of the left block is

$$
M_{b} \ddot{Y}_{\mathrm{Lbi}}=F_{L r L i}-F_{L s L i} .
$$

The equation of the vertical motion of the right block is

$$
M_{b} \ddot{Z}_{\mathrm{Rb} i}=F_{R r V i}-F_{R s V i} \text {. }
$$

The equation of the lateral motion of the right block is

$$
M_{b} \ddot{Y}_{\mathrm{Rb} i}=F_{R r L i}-F_{R s L i},
$$




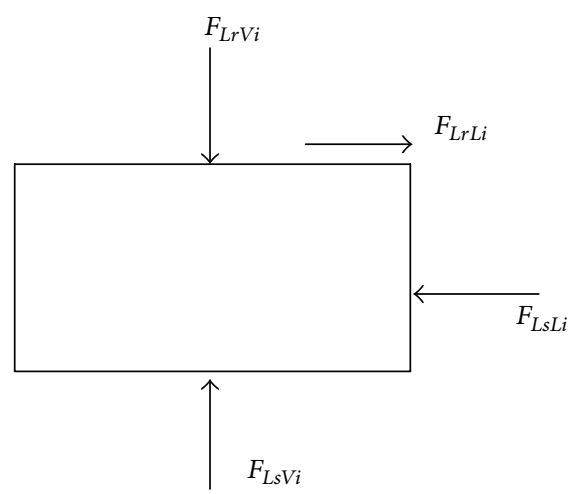

(a) The left block

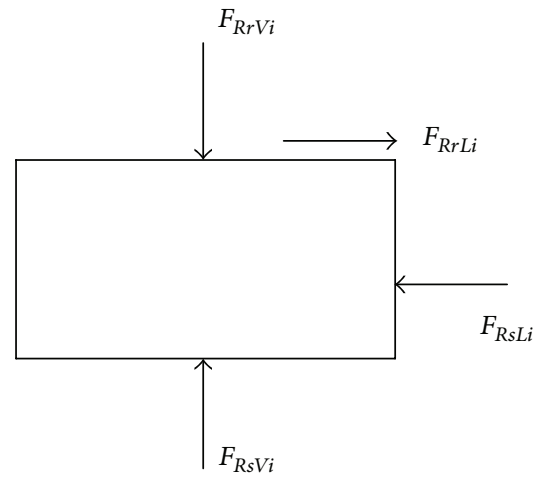

(b) The right block

FIGURE 5: Forces of the left and right block.

where $F_{L s V i}, F_{R s V i}, F_{L s L i}$, and $F_{R s L i}$ are the reaction forces of the concrete two-block sleeper:

$$
\begin{aligned}
& F_{L s V i}=K_{b v} Z_{\mathrm{Lb}}+C_{b v} \dot{Z}_{\mathrm{Lb}} \\
& F_{R s V i}=K_{b v} Z_{\mathrm{Rb}}+C_{b v} \dot{Z}_{\mathrm{Rb}} \\
& F_{L s L i}=K_{b h} Y_{\mathrm{Lb}}+C_{b h} \dot{Y}_{\mathrm{Lb}} \\
& F_{R s L i}=K_{b h} Y_{\mathrm{Rb}}+C_{b h} \dot{Y}_{\mathrm{Rb}}
\end{aligned}
$$

where $Z_{\mathrm{Lb}}$ and $Z_{\mathrm{Rb}}$ are the vertical displacements of the left and right block, respectively; $Y_{\mathrm{Lb}}$ and $Y_{\mathrm{Rb}}$ are the lateral displacement of the left and right block; $\dot{Z}_{\mathrm{Lb}}$ and $\dot{Z}_{\mathrm{Rb}}$ are the vertical velocities of the left and right block, respectively; $\dot{Y}_{\mathrm{Lb}}$ and $\dot{Y}_{\mathrm{Rb}}$ are the lateral velocities of the left and right block, respectively.

2.2.3. General Equations of Motion. All the above equations for the vehicle and track components are combined to form a set of ordinary differential equations expressed in terms of the following standard matrix form:

$$
[M]\{\ddot{X}(t)\}+[C]\{\dot{X}(t)\}+[K]\{X(t)\}=\{F(t)\},
$$

where $[M],[C]$, and $[K]$ are the generalized mass, damping, and stiffness matrices, respectively; $\{\ddot{X}(t)\},\{\dot{X}(t)\}$, and $\{X(t)\}$ are the generalized displacement, velocity, and acceleration vectors, respectively; and $\{F(t)\}$ is the corresponding force vector containing the wheel/rail forces $P_{j}(t)(j=1 \sim 4)$.

2.2.4. Initial and Boundary Conditions of the Coupled VehicleTrack System. The deflections and the bending moments at the beam-hinged ends are assumed to be zero. The deflection of the bed is ignored. The initial displacements and velocities of all components of the track are set to be zero. The initial displacements and the initial vertical and lateral velocities of all components of the vehicle are set to be zero, and the initial longitudinal velocity is the running speed of the vehicle, which is a constant.
2.2.5. Numerical Integration of the Coupled Vehicle-Track System. Due to the strong nonlinearities involved in the equations of motion of the large-scale coupled vehicle-track systems, the computation speed, accuracy, and stability of the numerical integration method are quite important when calculating the dynamic response of the coupled vehicletrack system. Zhai [13] proposed the "New Fast Numerical Integration Method" to specially solve the coupled dynamics equations of the railway vehicle and track. In this paper, Zhai's method is used to solve the equations of motion of the coupled vehicle-track system with a time step size of $1 \times 10^{-4} \mathrm{~s}$.

\section{Results and Discussion}

3.1. Influence of Voided Sleepers on Vehicle System. Due to the wear and damage of the rail, the nonuniformity of the sleeper span, the errors in the manufacture and installation of the fastener system, the stiffness changes in the pads, and other factors, the actual geometry of an urban rail line is usually random. In order to fully reflect the influence of voided sleepers of the elastic two-block sleeper track on the track system and the vehicle system, the vibration responses of the track and the vehicle to voided sleepers and the typical track random irregularity are compared. The measured random irregularity, strictly controlled by construction quality and the errors of the track, is chosen as the excitation, which is the vector irregularity of the rail tread at the weld joint and the vertical profile irregularity of the rail surface, measured by the $10 \mathrm{~m}$ chord-length method, and the corresponding irregularity peaks of $0.3 \mathrm{~mm}$ and $4 \mathrm{~mm}$, respectively. At present, there has been little research on the statistic features of the random irregularity of the urban transit railway, and there is no statistic random irregularity spectrum. So, the United States' railway's track irregularity spectrum is selected to simulate the track random irregularity of the urban transit railway in the present paper. The numerical simulation methods for various kinds of irregularities are described in [5]. In order to improve the computation efficiency, the vehicle concerned in this paper is the metro-A series train, and the vehicle speed $v$ is $60 \mathrm{~km} / \mathrm{h}$ in the numerical simulations. The parameters of the vehicle and track are given in [5]. 
TABLE 1: Comparison of vibration responses of vehicle system.

\begin{tabular}{lcccc}
\hline Index & Voided sleeper & Weld joint irregularity & Vertical profile irregularity & $\begin{array}{c}\text { American track } \\
\text { spectrum of fifth grade }\end{array}$ \\
\hline Peak of displacement of car body $(\mathrm{mm})$ & 1.9150 & 1.8127 & 3.3610 & 1.7340 \\
Peak of acceleration of car body $(\mathrm{g})$ & 0.00115 & 0.00125 & 0.00945 & 0.00906 \\
Peak of displacement of frame $(\mathrm{mm})$ & 2.059 & 1.850 & 4.722 & 1.653 \\
Peak of acceleration of frame $(\mathrm{g})$ & 0.00189 & 0.00246 & 0.01747 & 0.01361 \\
\hline
\end{tabular}

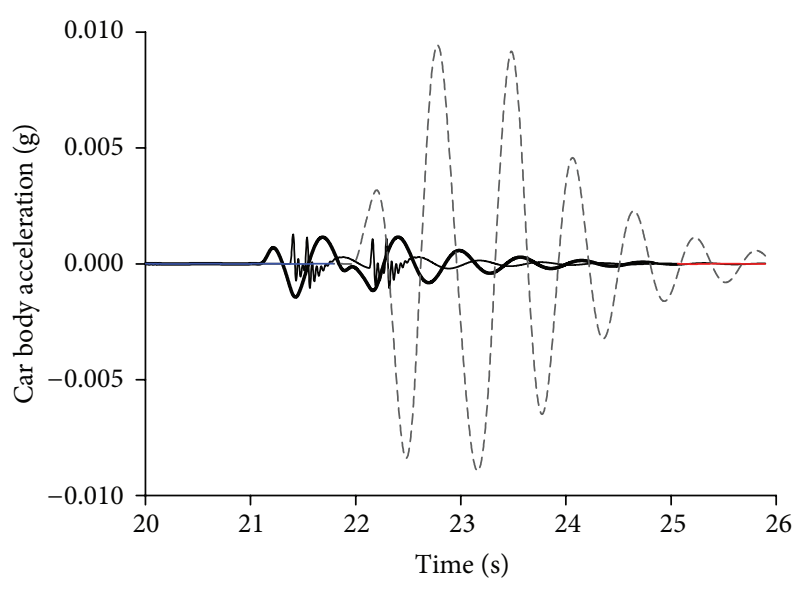

FIGURE 6: Vehicle acceleration corresponding to different stimulations. Bold line: voided sleepers; solid line: weld joint irregularity; dashed line: vertical profile irregularity.

The vehicle-track system is excited, respectively, by the geometric irregularity aforementioned and the stiffness irregularity caused by a single voided sleeper at the left side of the elastic two-block sleeper track. The time history of the vertical acceleration of the mass centre of the car body is obtained by the developed model, as shown in Figure 6. It can be seen that the car body acceleration, excited by the vertical profile irregularity of the rail surface, is far greater than that excited by the voided sleeper and weld joint irregularity. The acceleration peaks of car body excited by the voided sleeper and the weld joint irregularity are equivalent, but the waveform cycle of acceleration of the car body excited by the voided sleeper is longer.

The time history of the vibration acceleration of a car body excited by different numbers of voided sleepers is shown in Figure 7. Figure 7 shows that the acceleration is sensitive to the number of voided sleepers, and the acceleration peak arises rapidly with the increase in the number of voided sleepers. By comparing Figures 6 and 7, it can be seen that even if there are three sleepers voided continuously, the vibration acceleration peak of the car body is lower than that excited by the vertical profile irregularity of the rail surface.

The maximal peaks of vibration displacement and acceleration of the car body and the frames by the stiffness irregularity caused by a single voided sleeper under the left rail, the measured geometric irregularity, and the random irregularity are, respectively, listed in Table 1 . The data in the table show that the influences of the voided sleeper and

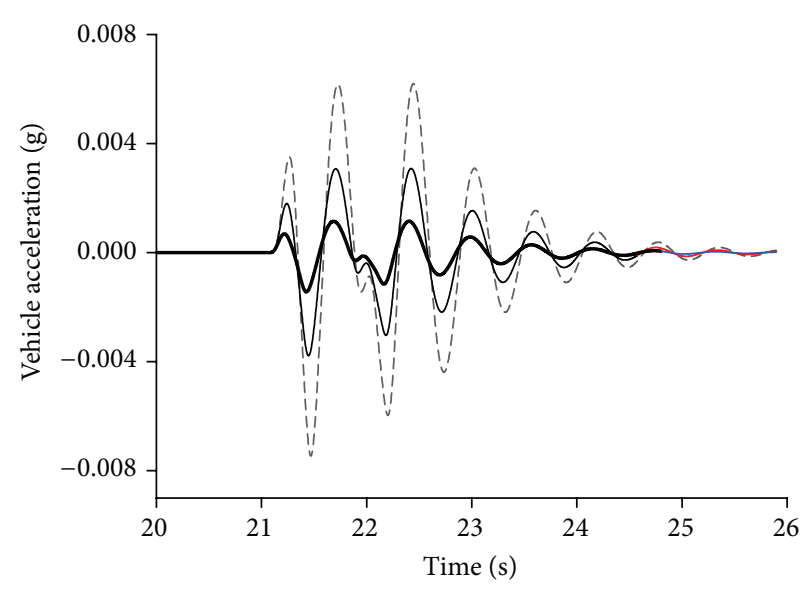

FIGURE 7: Vehicle acceleration corresponding to different numbers of voided elastic two-block sleepers. Bold line: one sleeper voided; solid line: two sleepers voided continuously; dashed line: three sleepers voided continuously.

the weld joint irregularity on the responses of the vehicle system are slightly different, and the influences of the voided sleeper are smaller; the vibration accelerations of the car body and the frame excited by both the vertical profile irregularity of the rail surface and the track random irregularity of the United States' railway are relatively larger; however, the difference between the two excitations is small; the vibration accelerations of the car body and frame excited by the track random irregularity of the United States' railway are, respectively, 8.9 times and 8.2 times that of those excited by the voided sleeper.

The direct influence of a single voided sleeper at one side of the track on the responses of the vehicle system is not obvious, compared to the measured geometric irregularity of the rail or the track random irregularity. So, it is not the factor affecting the vehicle running comfort and is not easily identified, until more sleepers on single side are voided continuously.

3.2. Influence of Voided Sleeper on Wheel/Rail Force. The wheel/rail force is an important evaluation index of dynamic interaction between the vehicle and the track. For the elastic two-block sleeper monolithic track, a sleeper under the left rail is assumed to be voided. Deducting the mean value of the wheel/rail force, the vertical and lateral wheel/rail force time history are, respectively, shown in Figures 8 and 9. Due to the effect of the voided sleeper, both the vertical and lateral wheel/forces have peak impact, and the impact peak of 


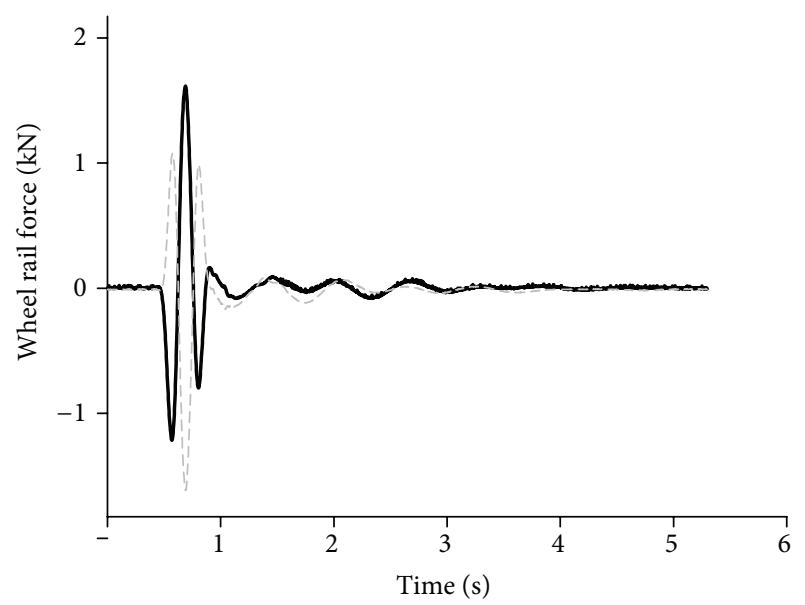

Figure 8: Vertical wheel/rail force (mean value is deducted). Bold line: vertical wheel/rail force of left side; dashed line: vertical wheel/rail force of right side.

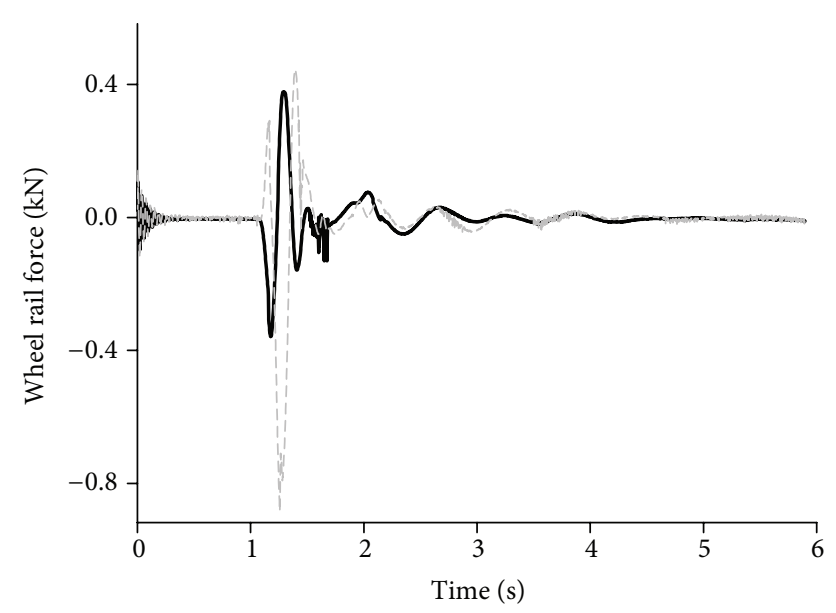

FIgURE 9: Lateral wheel/rail force (mean value is deducted). Bold line: lateral wheel/rail force of left side; dashed line: lateral wheel/rail force of right side.

the vertical wheel/rail force is far greater than that of the lateral wheel/rail force. So a single sleeper voided at one side has a greater effect on the vertical wheel/rail force than on the lateral wheel/rail force.

By comparing the left and right wheel/rail forces in Figures 8 and 9, it can be seen that although the sleeper is voided at the left of the track, the difference between the vertical and lateral wheel/rail forces is not obvious, and the amplitude of the right lateral wheel/rail force is larger than that of the left lateral wheel/rail force. At the same time, because of the poor bonding between elastic two-block sleeper and the monolithic track bed, the influence of the voided sleepers is very strong.

The wheel/rail forces excited by the weld joint irregularity, the vertical profile irregularity, and track random irregularity of the United States' railway are shown in Figure 10. The peaks of the vertical wheel/rail forces, from high to low, respectively, correspond to the weld joint irregularity, a voided sleeper,

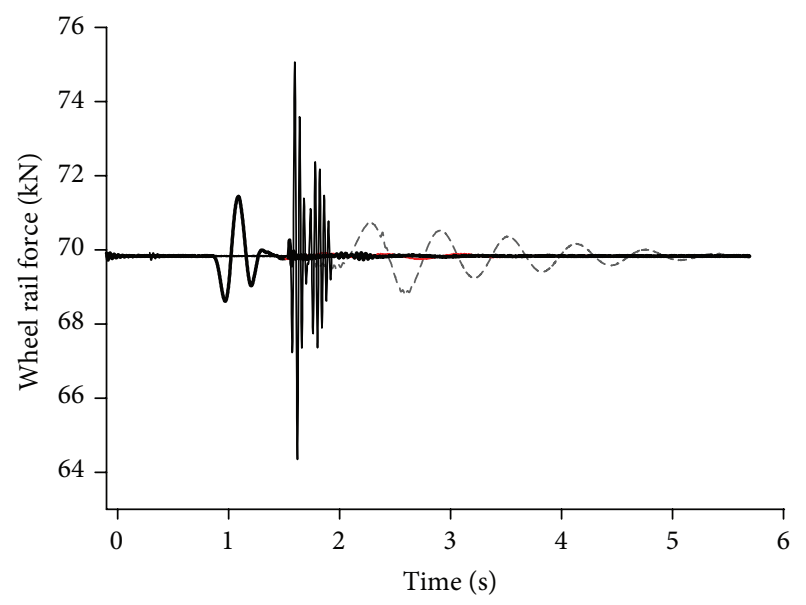

FIgURE 10: Vertical wheel/rail force corresponding to different excitations. Bold line: voided sleeper; solid line: weld joint irregularity; dashed line: sinusoidal excitation.

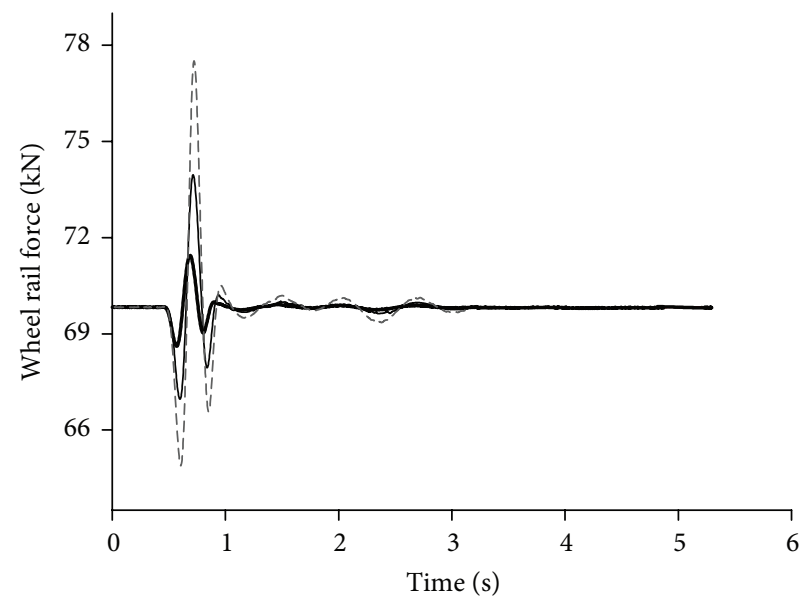

FIGURE 11: Vertical wheel/rail force corresponding to elastic twoblock sleeper continuously voided. Bold line: one sleeper voided; solid line: two sleepers continuously voided; dashed line: three sleepers continuously voided.

and sinusoidal excitation. The influence of the number of sleepers continuously voided under the left rail on the vertical wheel/rail force is shown in Figure 11. By comparing Figures 10 and 11 , it can be seen that if there are three sleepers continuously voided, the peak of the vertical wheel/rail force is far greater than that excited by the weld joint irregularity. Using the developed model, the maximal vertical wheel/rail force excited by the track random irregularity of the United States' railway is $74.7 \mathrm{kN}$, which is equivalent to that excited by the weld joint irregularity as shown in Figure 10, and is $3.8 \%$ smaller than that excited by three sleepers continuously voided. Therefore, in theory, only when there are three or more sleepers voided at one side can the change of vertical wheel/rail force be detected.

3.3. Influence of Voided Sleeper on the Adjacent Sleepers. The voided sleeper has a great effect on the fasteners and 


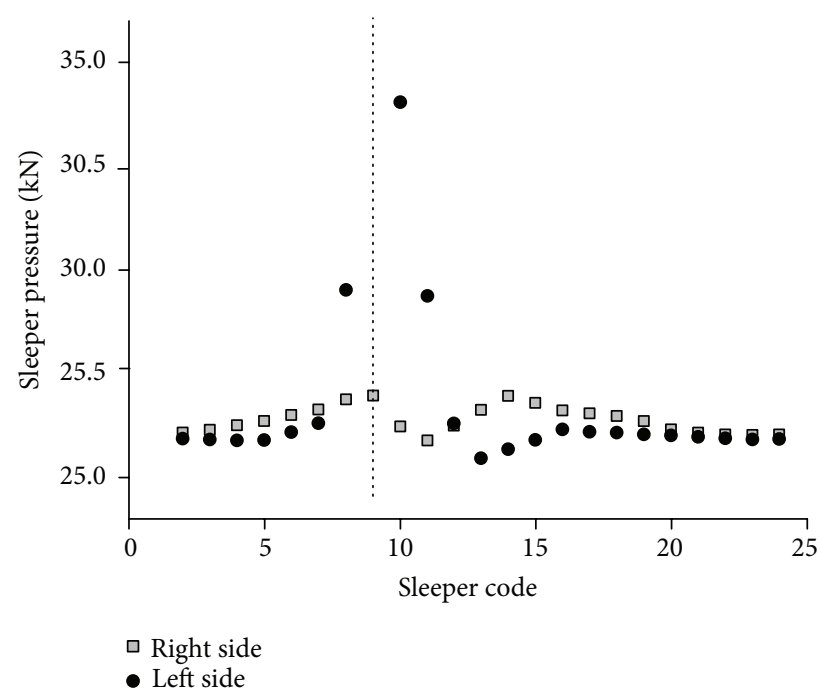

FIGURE 12: Sleeper pressure caused by one-side voided sleeper.

the adjacent sleepers, which can be evaluated by the pressures on the sleeper. When the ninth sleeper voided under the left rail is a voided sleeper, the maximal values of the pressures on the adjacent sleepers are shown in Figure 12. It is obvious that the pressures on the eighth, the tenth, and the eleventh sleepers increase significantly; in particular, the pressure on the tenth sleeper increases by $10 \mathrm{kN}$. The sleepers voided under the left rail have little effect on the pressure on the sleeper under the right rail.

When the ninth sleeper is voided under the two different conditions (voided at the left side only or on both sides), the pressures on the sleeper under the left rail are shown in Figure 13. It can be seen that, under the two different conditions, the influence of voided sleepers on the pressure on the sleeper is almost the same, the pressure on the tenth sleeper is the highest, and the pressures on the eighth and the eleventh sleepers are the next highest. But, under the condition of the ninth sleeper voided only under the left rail, the pressures on the eighth, the tenth, and the eleventh sleepers under the left rail are $0.3 \sim 0.4 \mathrm{kN}$ higher than that under the condition of the ninth sleeper voided under both rails. Therefore, compared with the sleeper voided under both rails, the sleeper voided under one side only has a greater effect on the pressure on the sleeper.

The influence of the number of elastic two-block sleepers continuously voided under the left rail on the pressures on the sleeper under the left rail is shown in Figure 14, which illustrates that the law of the pressure change of the adjacent sleeper caused by the sleepers continuously voided is consistent with that caused by a single voided sleeper. With the number of the sleepers continuously voided increasing, the pressure on the adjacent sleeper is higher. From Figure 14, it can be seen that the maximal pressures on a sleeper caused by a single sleeper and two and three sleepers continuously voided are found at the next adjacent sleeper, being $34 \mathrm{kN}, 45 \mathrm{kN}$, and $61 \mathrm{kN}$, respectively. The fastener pressure is commonly designed at about $40 \mathrm{kN}$, so the pressures on the sleeper caused by a single elastic voided

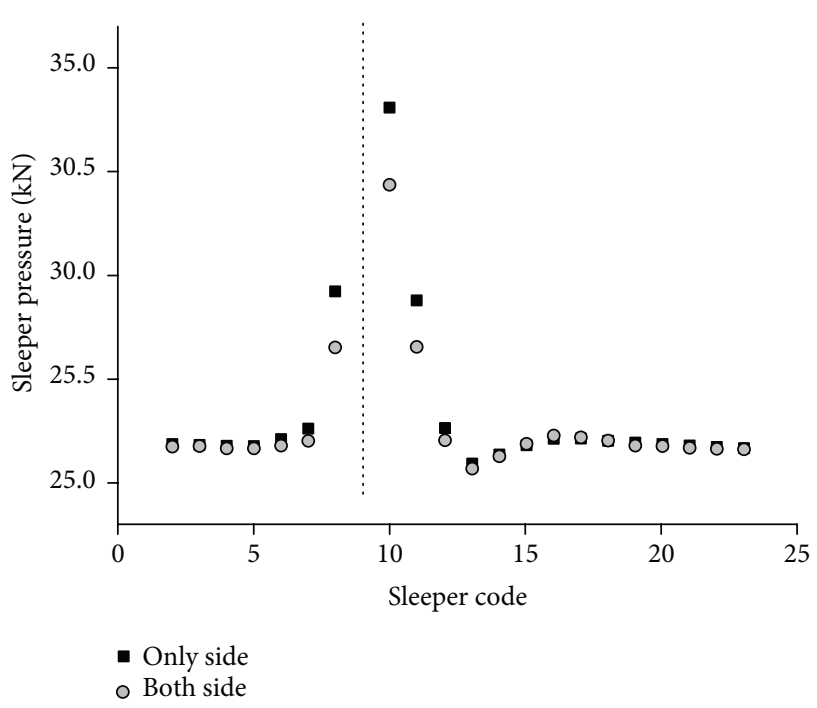

FIgURE 13: Pressures on sleeper corresponding to different conditions of voided sleeper.

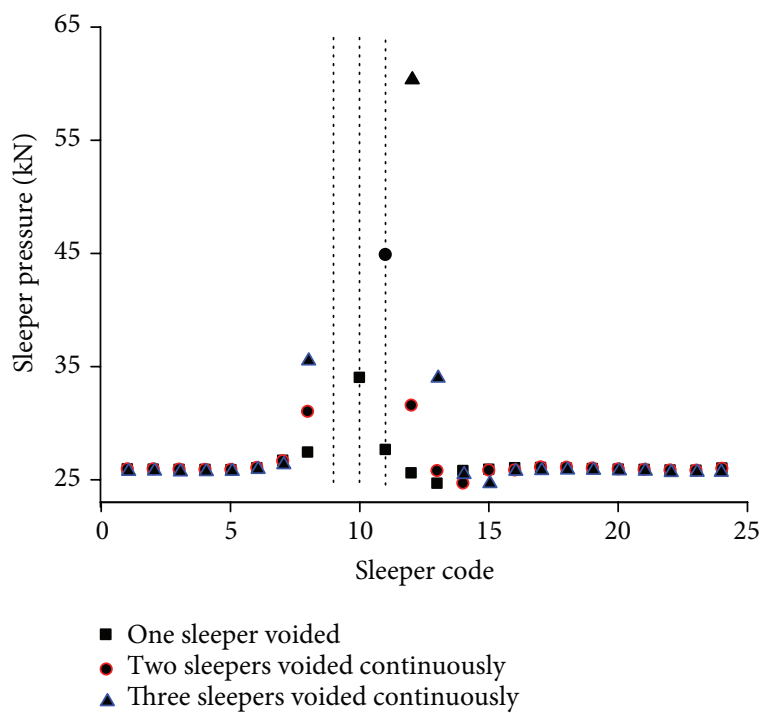

FIGURE 14: Effect of continuous voided sleepers on the sleeper pressure.

sleeper will theoretically satisfy the fastener pressure design requirement, but the pressures on the sleeper caused by two and more sleepers continuously voided will go beyond the design limits of the fastener pressure.

When a single elastic two-block sleeper is voided under the left rail, the influence of different train speeds on the maximal pressure on the sleeper under the left rail is shown in Figure 15. From Figure 15, it can be seen that the relationship between the maximum pressure value and the train speed is approximately linear. The maximum pressure value increases by only about $0.14 \mathrm{kN}$ with each $20 \mathrm{~km} / \mathrm{h}$ increase in train speed; the sleeper pressure increases by about $0.56 \mathrm{kN}$ when the train speed rises from $40 \mathrm{~km} / \mathrm{h}$ to $120 \mathrm{~km} / \mathrm{h}$. So, for the urban railway with a maximum design speed of $120 \mathrm{~km} / \mathrm{h}$, it can be considered that the train speed has little effect on 
TABLE 2: Comparison of maximum sleeper pressures (kN).

\begin{tabular}{lccccc}
\hline Index & No irregularity & Vertical irregularity & Weld irregularity & American track spectrum & A single voided sleeper \\
\hline Maximum sleeper pressure & 25.96 & 26.81 & 27.43 & 29.05 & 33.54 \\
\hline
\end{tabular}

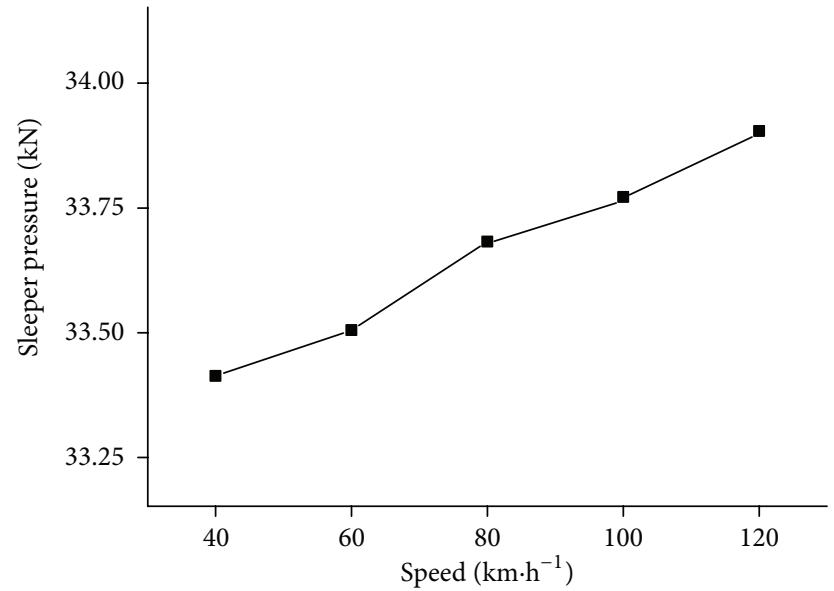

FIGURE 15: Effect of vehicle speed on the sleeper pressure.

the maximal pressures on sleeper change caused by elastic two-block voided sleepers.

The maximal sleeper pressures caused by different track irregularities are listed in Table 2. The vertical profile irregularity, weld joint irregularity, track random irregularity of the United States' railway, and a single voided sleeper have linearly increasing influence on the maximal pressures on the sleeper. But the maximum pressure caused by a single voided sleeper is far greater than that caused by other irregularities.

\section{Conclusions}

Based on vehicle-track coupled dynamic theory, a threedimensional asymmetric vehicle-track coupling vibration model is developed to investigate the effect of voided elastic two-block sleeper on vehicle and track system dynamic responses. The Hertz nonlinear elastic contact theory is used to calculate the normal wheel/rail force. The wheel/rail tangent creep force is first calculated using Kalker's linear creep theory and then modified by the Shen-Hedrick-Elkins theory. Some conclusions are as follows:

(1) A single voided elastic two-block sleeper has the most obvious effect on the pressures on the sleeper, followed by the wheel/rail force, but has a very limited effect on the comfort of the car body.

(2) For an urban railway with a low train speed, the pressure on a sleeper caused by it being a single voided sleeper is greater than that caused by the vertical profile irregularity, weld joint irregularity, or track random irregularity of the United States' railway, but the pressures on the sleeper caused by only a single voided elastic sleeper still meet the design limits of the fastener pressure. However, in the case of two and more sleepers continuously voided, the pressures on the sleeper will go beyond the design limits of the fastener pressure, which may be harmful to the safe operation of the train.

(3) The effects on the pressures on the sleeper, of the sleeper voided at one side and both sides, are nearly the same. But, compared to sleepers voided on both sides, the effect of a sleeper voided at one side has a slightly greater effect on the maximal pressure on the sleeper.

(4) The sleeper voided at one side makes the vertical and lateral wheel/rail forces increase, with the vertical wheel/rail force increase being greater. By comparison, it can be seen that only when there are three or more sleepers voided at one side can this be detected by the change in the vertical wheel/rail force, but, at this time, both the fastener pressure and the pressure on the sleeper are beyond the design limits.

(5) The voided sleepers have little effect on the vibration of the vehicle system. Even if there are three sleepers continuously voided, the vibration acceleration excitation peaks are lower than those excited by the vertical profile irregularity. Therefore, the voided sleepers are not easily identified by the vibration response of the vehicle system, and the track inspection should be enhanced during operation.

\section{Conflict of Interests}

The authors declare that there is no conflict of interests regarding the publication of this paper.

\section{Acknowledgments}

This research was supported by the National Natural Science Foundation of China (no. 51165017 and no. 51378395) and the Fundamental Research Funds for the Central Universities (Tongji University).

\section{References}

[1] E. L. Olsson and P. Zackrisson, Long-Term Measurement Results, Swedish National Road Administration, Borlänge, Sweden, 2002.

[2] S. Auguetin, G. Gudehus, G. Huber, and A. Schünemann, "Numerical model and laboratory tests on settlement of ballast track," in System Dynamics and Long-Term Behaviour of Railway Vehicles, Track and Subgrade, vol. 6 of Lecture Notes in Applied Mechanics, pp. 317-336, Springer, Berlin, Germany, 2003.

[3] S. L. Grassie and S. J. Cox, "Dynamic response of railway track with unsupported sleepers," Transport Engineering, vol. 199, no. 2, pp. 123-135, 1985. 
[4] M. Ishida, T. Moto, A. Kono, and Y. Jin, "Influence of loose sleeper on track dynamics and bending fatigue of rail welds," Quarterly Report of RTRI, vol. 40, no. 2, pp. 80-85, 1999.

[5] W. M. Zhai, Vehicle-Track Coupled Dynamics, Science Press, Beijing, China, 3rd edition, 2007 (Chinese).

[6] C.-H. Zou, S.-H. Zhou, and B.-L. Wang, "Calculation method of unsupported sleepers caused by differential subgrade settlement of ballasted track," Journal of the China Railway Society, vol. 35, no. 1, pp. 87-92, 2013 (Chinese).

[7] Z. H. He, "The dynamic influences of the sleeper gap in urban rail transit," Railway Engineering, vol. 3, pp. 86-88, 2010 (Chinese).

[8] J. Xiang, H. Yang, and D. He, "Research on the responses of vertical vibration of train-track system due to voided sleepers," Journal of Railway Science and Engineering, vol. 4, no. 1, pp. 8-12, 2007 (Chinese).

[9] A. Z. Jabbar, H. Xia, and J. J. Fan, "Effects of unsupported sleeper on dynamic responses of railway track," Journal of Northern Jiaotong University, vol. 24, no. 1, pp. 50-55, 2000.

[10] X. B. Xiao, X. S. Jin, and Z. F. Wen, "Effect of hard spot on dynamic response of railway sleeper," Journal of Traffic and Transportation Engineering, vol. 24, no. 1, pp. 50-55, 2000 (Chinese).

[11] J. Zhang, C. H. Wu, X. B. Xiao, Z. Wen, and X. Jin, "Effect of unsupported sleepers on sleeper dynamic response," Journal of Southwest Jiaotong University, vol. 45, no. 2, pp. 203-208, 2010 (Chinese).

[12] Z. Y. Shen, J. K. Hedrick, and J. A. Elkins, "A comparison of alternative creep force models for rail vehicle dynamic analysis," in Proceedings of the 8th IAVSD Symposium, pp. 591605, Cambridge, Mass, USA, January 1984.

[13] W.-M. Zhai, "Two simple fast integration methods for largescale dynamic problems in engineering," International Journal for Numerical Methods in Engineering, vol. 39, no. 24, pp. 41994214, 1996. 


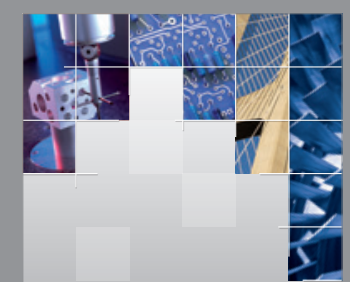

\section{Enfincering}
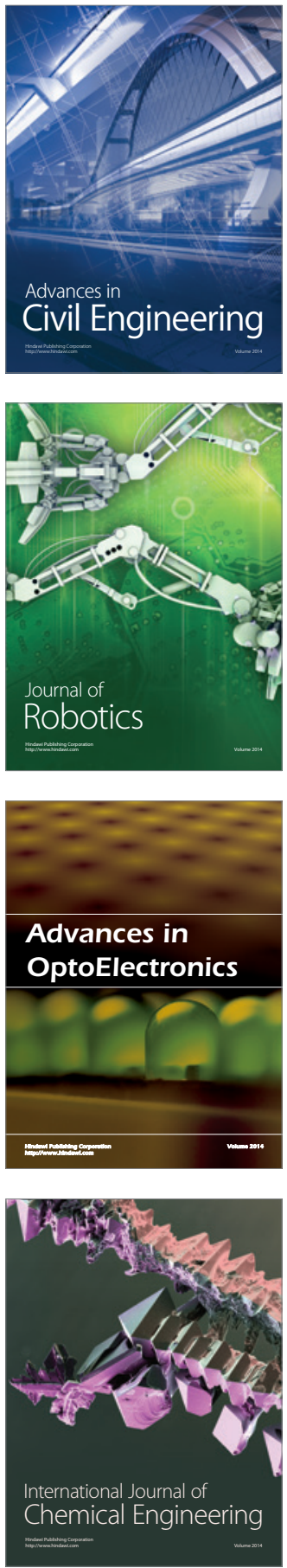

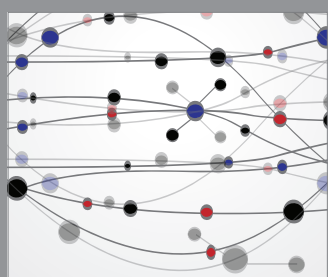

The Scientific World Journal

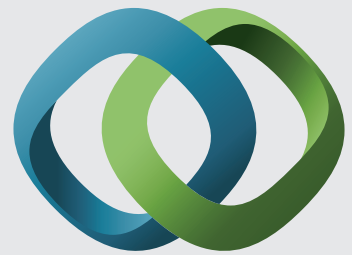

\section{Hindawi}

Submit your manuscripts at

http://www.hindawi.com
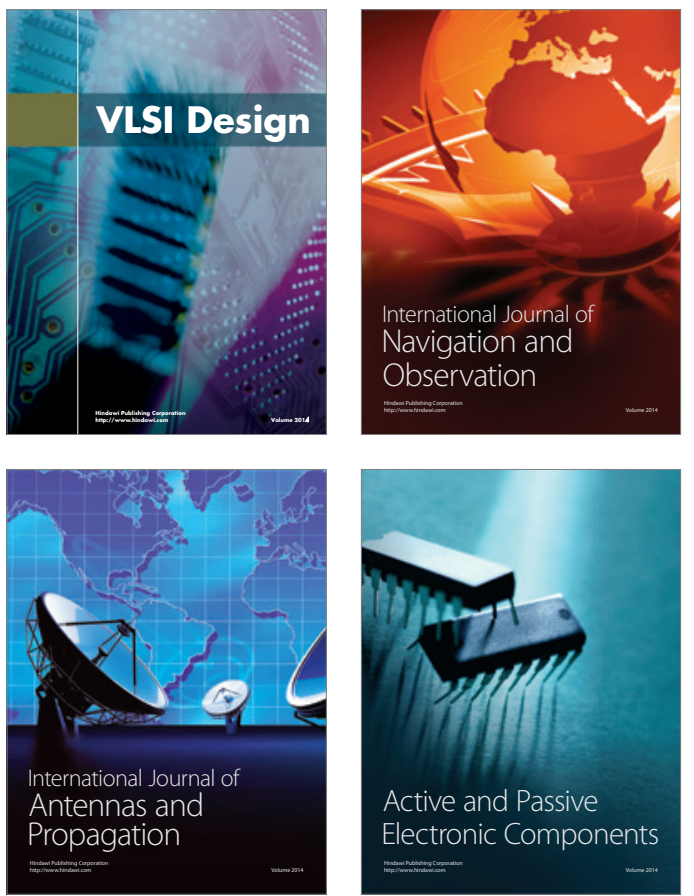
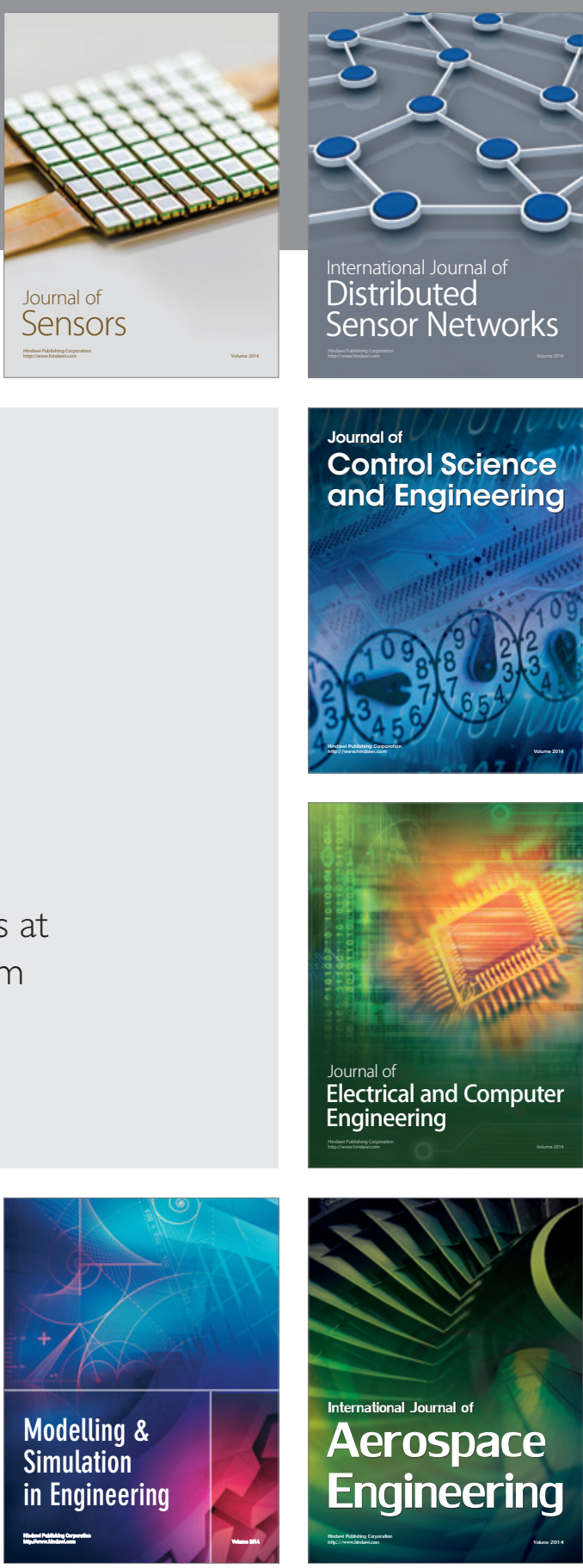

International Journal of

Distributed

Sensor Networks

Journal of

Control Science

and Engineering
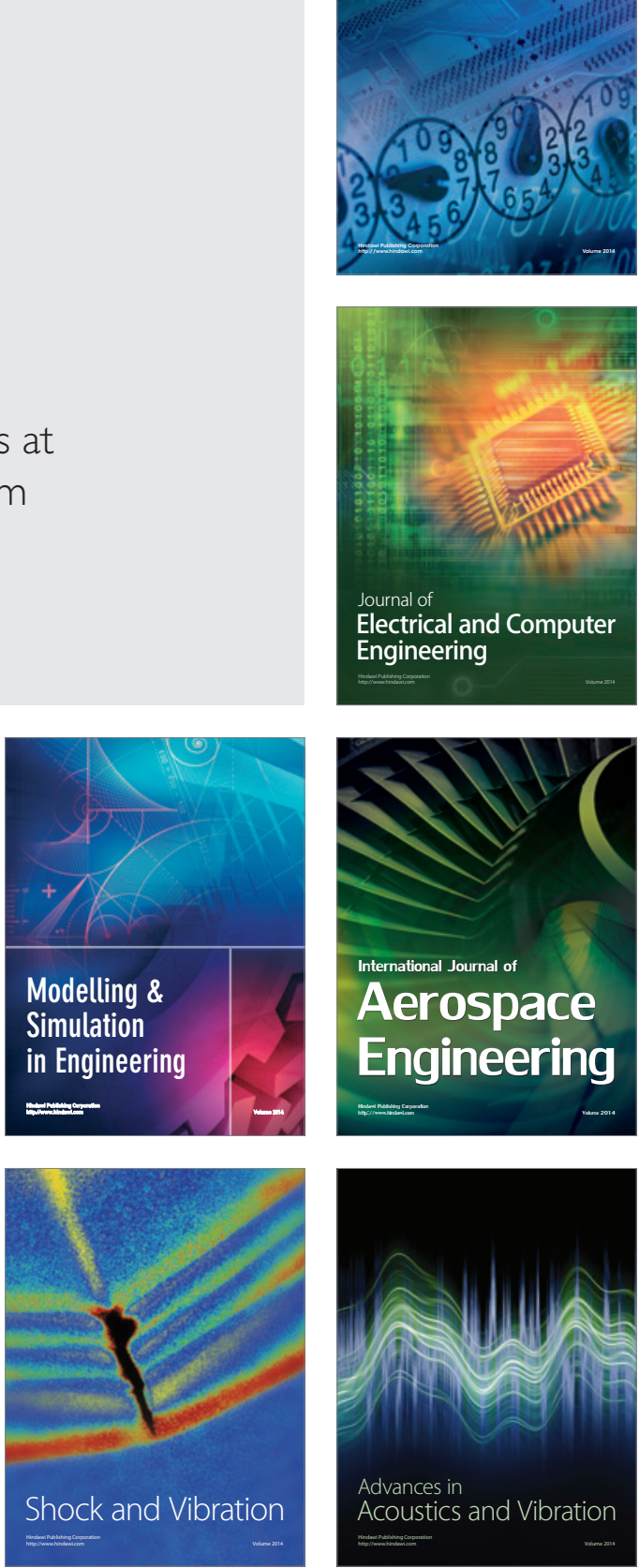